\title{
Attack and Improvement of a Proxy Multi-Signature Scheme
}

\author{
WEI Hong-ru, HU Jia-yuan* \\ School of Mathematics and Physics \\ University of Science and Technology Beijing \\ Beijing 100083, China \\ e-mail: 837659172@qq.com
}

\begin{abstract}
Proxy multi-signature system is based on digital signature scheme for a class of special signature scheme, which requires the signer to own certain powers were delegated to each proxy signer to make these proxy signer to use these powers on behalf of himself. In this paper, a class before the enhanced proxy multi-signature scheme carry out the attack and improvement, three forgery attacks has been implemented,one for the any original signer,one for the any proxy signer,and one for the proxy signer modify the proxy deadline. While the original proposals put forward an improved model, to prevent a proxy signer rights transfer, the ability to prevent abuse of the signature to ensure that the proxy signature security.
\end{abstract}

Keywords-digital signature; proxy signature; proxy multisignature; forgery attack

\section{INTRODUCTION}

In recent years, with growing social networking technologies developed in the field of e-commerce, people often need to delegate some of their powers to give a reliable agent, agent on behalf of themselves and let them to exercise these powers. These can be delegated powers to include people in the right signature in 1996,Mambo, Usuda and Okamoto[1], who proposed the concept of proxy signature, digital signature gives a solution to this problem proxy a solution method. Because proxy signature plays an important role in practical applications, so it was put forth proxy signature has been widespread concern, its scholars have also conducted in-depth discussion and research[2,3]. Since then, Elly river[4,5], who proposed the idea of proxy multi-signature, which allows multiple users to use proxy signature on the file signature process, in many areas of e-commerce, elections, protocol exchange has played an important role[6].

By studying Zhou Hongsheng, iron Ling, Li Jianhua, Niyou Sheng et al[7] article, the second proxy multi-signature scheme given its paper analyzed, and any one of the original signers, any proxy signer, proxy signer modify the proxy deadline forgery attack, he explained the existence of security issues. After its signing process given signature generation process and the commission process has been improved. And described in the program to improve the complexity of its calculation has reached the security requirements can withstand before the attacks.

\section{ZhOU HONGSHENG ET AL [7] PROXY MULTI-SIGNATURE SCHEME}

\section{A. Parameter Description}

$p$ is a large prime number, $q$ of $p-1$ or $p-1$ 's large prime factor, $g \in Z_{q}^{*}$ and $g^{q} \equiv 1(\bmod p) ; A_{i}$ is $L$ user digital signature scheme, $x_{A_{i}}$ is a secret key of the user $A_{i}, x_{A_{i}} \in Z_{q}^{*}, y_{A_{i}}$ for the user's public key $A_{i}, y_{A_{i}}=g^{x_{A_{i}}}(\bmod p) ; B_{i}$ is a digital signature scheme another $L$ users; secret key all proxy signers $B_{i}$ is, $x_{B_{i}} \in Z_{q}^{*}$ the public key is $y_{B_{i}}=g^{x_{B_{i}}}(\bmod p) ; h()$ is a hash function; $w_{i}$ commission is to delegate authority $A_{i}$ to $B_{i}$ a signature file.

\section{B. commissioning process}

1) $A_{i}$ randomly selected for each user a number $k_{i} \in Z_{q}^{*}$,

Calculate: $K_{i}=g^{k_{i}}(\bmod p), e_{i}=x_{A_{i}} h\left(w_{i}, K_{i}\right)+k_{i}(\bmod q)$;

2) $g^{e_{i}}=y_{A_{i}}{ }^{\mathrm{h}\left(\mathrm{w}_{i}, \mathrm{~K}_{i}\right)} \mathrm{K}_{i}(\bmod p)$ verification is established.

\section{Signature generation}

1)Each agent signer $B_{i}$ randomly picks a number $r \in Z_{q}^{*}$, calculation: $R_{i}=g^{r_{i}}(\bmod p)$;

2)calculation:

$$
R=\prod_{i=1}^{L} R_{i}(\bmod p) s_{i}=\left(e_{i}-x B_{i}\right) m-n R(\bmod q) ;
$$

3)calculation: $R=\prod_{i=1}^{L} R_{i}(\bmod p)$,

test equation is established:

$$
\begin{aligned}
& \qquad\left(y_{A_{i}}{ }^{h\left(w_{i}, K_{i}\right)} K_{i}\right)^{m}=g^{s_{i}} R_{i}^{R} y_{B_{i}}{ }^{m}(\bmod p), \\
& \text { calculation: } s=\sum_{i=1}^{L} s_{i}(\bmod q),
\end{aligned}
$$

The message of the proxy multi-signature is :

$\left(R ; K_{1}, K_{2}, \ldots, K_{L} ; w_{1}, w_{2}, \ldots w_{L} ; s ; y_{A_{1}}, y_{A_{2}}, \ldots y_{A_{L}} ; y_{B_{1}}, y_{B_{2}}, \ldots, y_{B_{L}}\right)$. 


\section{PAIRS OF 2 PROXY MULTI-SIGNATURE ATTACKS}

\section{A. pairs of any original signer forgery attack}

For any who want to forge the original signature of the signer, may wish to set $A_{0}$, want to take any message $m^{\prime}$ to generate a valid proxy multi-signature:

$$
\left(R, S^{\prime}, K_{1}^{\prime}, K_{2}, \ldots, K_{L}, w_{i}, y_{A_{i}}, y_{B_{i}}\right)(i=1,2, \ldots, L),
$$

then perform the following steps:

1)Calculation: $d=\mathrm{m} \cdot\left(\mathrm{m}^{\prime}\right)^{-1}(\bmod q)$.

2)Calculation:

$$
K_{1}{ }^{\prime}=\left(\prod_{i=1}^{L}\left(y_{A_{i}}{ }^{h\left(W_{i}, K_{i}\right)} K_{i}\right)\right)^{d-1} y_{A_{0}}{ }^{h\left(W_{1}, K_{1}\right)} \cdot K_{1}\left(\prod_{i=1}^{l} y_{B_{i}}\right)^{1-d}(\bmod p) \text {. }
$$

3)Calculation: $S^{\prime}=S+x_{A_{1}} \mathrm{~h}\left(\mathrm{w}_{1}, \mathrm{k}_{1}{ }^{\prime}\right) \mathrm{m}^{\prime}(\bmod q)$.

The proxy multi-signature:

$$
\left(R, S^{\prime}, K_{1}^{\prime}, K_{2}, \ldots, K_{L}, w_{i}, y_{A_{i}}, y_{B_{i}}\right)(i=1,2, \ldots, L)
$$

can verify the person's verification,Proof:

$$
\begin{aligned}
& \left.\left\{\left(\mathrm{y}_{A_{0}}{ }^{\mathrm{h}\left(w_{1}, k_{1}\right)} K_{1}^{\prime}\right) K_{1}^{\prime}\right) \prod_{i=2}^{L}\left(y_{A_{i}}{ }^{h\left(w_{i}, K_{i}\right)} K_{i}\right)\right\}^{m^{\prime}} \\
& =\left\{y_{A_{0}} h\left(w_{1}, k_{1}^{\prime}\right) \prod_{i=1}^{L}\left(y_{A_{i}}{ }^{h\left(w_{i}, K_{i}\right)}\right)\right\}^{d-1} \\
& =y_{A_{0}}{ }^{h\left(w_{1}, k_{1}\right)} \cdot K_{1}\left[\prod_{i=1}^{L} y_{B_{i}}\right]^{1-d} \prod_{i=2}^{L}\left(y_{A_{i}}{ }^{h\left(w_{i}, K_{i}\right)}\right)^{m^{\prime}} \\
& =\left(g^{x} A_{0}^{h\left(w_{1}, K_{1}\right)}\right) m^{\prime} \cdot\left[\prod_{i=1}^{L}\left(y_{A_{i}}{ }^{h\left(w_{i}, K_{i}\right)} K_{i}\right)\right]^{m} \cdot\left[\left(\prod_{i=1}^{L} y_{B_{i}}\right)^{1-d}\right]^{m^{\prime}} \\
& =\left(g^{x} A_{0}^{h\left(w_{1}, K_{1}{ }^{\prime}\right)}\right)^{\mathrm{m}^{\prime}+s} R^{R}\left[\left(\prod_{i=1}^{L} y_{B_{i}}\right)^{d m^{\prime}}\right. \\
& =\left[\left(\prod_{i=1}^{L} y_{B_{i}}\right)^{1-d}\right]^{m^{\prime}}=\mathrm{g}^{\mathrm{S}^{\prime}} R^{R}\left(\prod_{i=1}^{l} y_{B_{i}}\right)^{m^{\prime}(\bmod p) .}
\end{aligned}
$$

\section{B. pairs of any proxy signer forgery attack}

Any one want to forge proxy signer signer may wish to want to take any message to generate a valid proxy multisignature:

$$
\left(R, S, y_{B_{1}}^{\prime}, y_{B_{2}}, \ldots, y_{B_{L}}, K_{i}, w_{i}, y_{A_{i}}\right)(i=1,2, \ldots, L)
$$

after performing the following steps:

1)Calculation: $d=\mathrm{m} \cdot\left(\mathrm{m}^{\prime}\right)^{-1}(\bmod q)$.

2)Calculation:

$$
y_{B_{0}}{ }^{\prime}=\left(\prod_{i=1}^{L}\left(y_{A_{i}}{ }^{h\left(W_{i}, K_{i}\right)} K_{i}\right)\right)^{d-1} y_{B_{0}} \cdot\left(\prod_{i=1}^{l} y_{B_{i}}\right)^{\mathrm{d}-1}(\bmod p) .
$$

After $B_{0}$ can own private key is revealed by his own public key to $y_{B_{0}}{ }^{\prime}$, the proxy multi-signature:

$$
\left(R, S, y_{B_{1}}^{\prime}, y_{B_{2}}, \ldots, y_{B_{L}}, K_{i}, w_{i}, y_{A_{i}}\right)(i=1,2, \ldots, L)
$$

can verify the person's verification,proof:

$$
\begin{aligned}
& g^{S} R^{R}\left(y_{B_{0}}{ }^{\prime} \prod_{i=2}^{L} y_{B_{i}}\right)^{\mathrm{m}} \\
& =g^{S} R^{R}\left\{\left[\left(\prod_{i=1}^{L}\left(y_{A_{i}}{ }^{h\left(w_{i}, K_{i}\right)} K_{i}\right)\right)^{1-\mathrm{d}} \mathrm{y}_{B_{0}}\left(\prod_{i=1}^{L} y_{B_{i}}\right)^{d-1}\right] \prod_{i=2}^{L} y_{B_{i}}\right\}^{m^{\prime}} \\
& \left.=g^{S} R^{R}\left(\prod_{i=1}^{L} y_{B_{i}}\right)^{d \mathrm{~m}^{\prime}}\left\{\left[\prod_{i=1}^{L}\left(y_{A_{i}}{ }^{h\left(w_{i}, K_{i}\right)} K_{i}\right)\right]^{1-d}\right]\right\}^{m^{\prime}} \\
& \left.\left.=\left[\prod_{i=1}^{L} y_{A_{i}}{ }^{h\left(w_{i}, K_{i}\right)} K_{i}\right)\right]^{\mathrm{m}}\left\{\left[\prod_{i=1}^{L}\left(y_{A_{i}}{ }^{h\left(w_{i}, K_{i}\right)} K_{i}\right)\right]^{1-d}\right]\right\}^{m^{\prime}} \\
& \left.=\left[\prod_{i=1}^{L} y_{A_{i}}{ }^{h\left(w_{i}, K_{i}\right)} K_{i}\right)\right]^{\mathrm{m}}(\bmod p) .
\end{aligned}
$$

\section{C. pairs proxy signer modify the proxy deadline forgery} attack

For any who want to forge a signature by modifying the term of agency, it may be set to $C_{0}$, you want to generate a valid proxy for any period by modifying agents take the message $m$ ' multi-signature:

$$
\left(R, S, y_{C_{1}}^{\prime}, y_{C_{2}}, \ldots, y_{C_{L}}, K_{i}, w_{i}{ }^{\prime}, y_{A_{i}}\right)(i=1,2, \ldots, L),
$$

he can perform the following steps:

1)Calculation: $d=\mathrm{m} \cdot\left(\mathrm{m}^{\prime}\right)^{-1}(\bmod q)$.

2)Calculation: $d_{i}=h\left(w_{i}, K_{i}\right) h\left(w_{i}{ }^{\prime}, K_{i}\right)(\bmod q)$, where $w_{i}{ }^{\prime}$ is the agent deadline modified value.

3)Calculation:

$$
\begin{aligned}
& y_{C_{0}}{ }^{\prime}=\left[\prod_{i=1}^{L}\left(y_{A_{i}}{ }^{h\left(W_{i}^{\prime}, K_{i}\right)} K_{i}\right)\right]^{d-1} \cdot \\
& \prod_{i=1}^{l}\left(y_{A_{i}}{ }^{\left(d_{i}-1\right) h\left(w_{i}^{\prime}, K_{i}\right)}\right)^{-d} y^{c_{0}}\left(\prod_{i=1}^{L} y_{c_{i}}\right)^{d-1}(\bmod p) .
\end{aligned}
$$

After $C_{0}$ can be your own private key is revealed by his own public key to $y_{C_{0}}{ }^{\prime}$, then the proxy multi-signature:

$$
\left(R, S, y_{C_{1}}^{\prime}, y_{C_{2}}, \ldots, y_{C_{L}}, K_{i}, w_{i}{ }^{\prime}, y_{A_{i}}\right)(i=1,2, \ldots, L) \text {, }
$$

person can be verified by the verification, prove: 


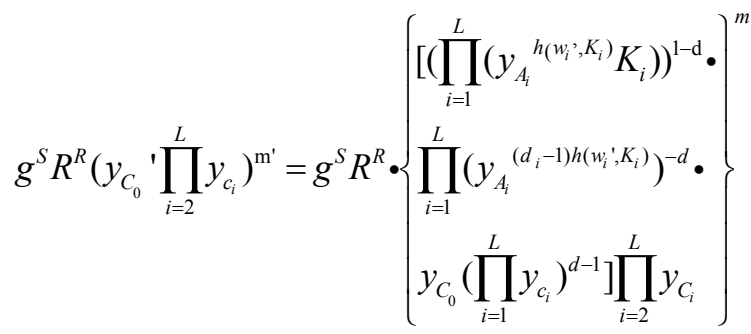

$$
\begin{aligned}
& =g^{S} R^{R}\left(\prod_{i=1}^{L} y_{C_{i}}\right)^{d \mathrm{~m}^{\prime}} \cdot\left\{\begin{array}{l}
{\left[\prod_{i=1}^{L}\left(y_{A_{i}}{ }^{h\left(w_{i}^{\prime}, K_{i}\right)} K_{i}\right)\right]^{1-d} \cdot} \\
\prod_{i=1}^{L}\left(y_{A_{i}}{ }^{\left(d_{i}-1\right) h\left(w_{i}, K_{i}\right)}\right)^{-d}
\end{array}\right\} \\
& \left.=\left[\prod_{i=1}^{L} y_{A_{i}}{ }^{h\left(w_{i}, K_{i}\right)} K_{i}\right)\right]^{\mathrm{m}} \cdot\left\{\begin{array}{l}
{\left[\prod_{i=1}^{L}\left(y_{A_{i}}{ }^{h\left(w_{i} ; K_{i}\right)} K_{i}\right)\right]^{1-d} \cdot} \\
\prod_{i=1}^{L}\left(y_{A i}{ }^{\left(d_{i}-1\right) h\left(w_{i} ; K_{i}\right)}\right)^{-d}
\end{array}\right\} \\
& \left.=\left[\prod_{i=1}^{L} y_{A_{i}} d_{i} h\left(w_{i}^{\prime}, K_{i}\right) K_{i}\right)\right]^{\mathrm{dm} \cdot} \cdot\left\{\begin{array}{l}
{\left[\prod_{i=1}^{L}\left(y_{A_{i}}{ }^{h\left(w_{i} ; K_{i}\right)} K_{i}\right)\right]^{1-d} \cdot} \\
\prod_{i=1}^{L}\left(y_{A_{i}}{ }^{\left(d_{i}-1\right) h\left(w_{i}^{\prime}, K_{i}\right)}\right)^{-d}
\end{array}\right\} \\
& \left.=\left[\prod_{i=1}^{L} y_{A_{i}}{ }^{h\left(w_{i}^{\prime} ; K_{i}\right)} K_{i}\right)\right]^{\mathrm{m}}(\bmod p) \text {. }
\end{aligned}
$$

\section{IMPROVEMENT AND PROXY MULTI-SIGNATURE ATTACK IN 2}

We conducted three types of attack in 3, found 2 Zhou Hongsheng et al proxy multi-signature scheme is not safe, we will improve its programs:

\section{1) delegate process}

Each user $A_{i}$ randomly selected a number $k_{i} \in Z_{q}^{*}$, calculated:

$$
K_{i}=g^{k_{i}}(\bmod p), e_{i}=x_{A_{i}} h\left(w_{i}, K_{i}, y_{A_{i}}\right)+k_{i} K_{i}(\bmod q) ;
$$

Verify: $g^{e_{i}}=y_{A_{i}}{ }^{\mathrm{h}\left(\mathrm{w}_{i}, \mathrm{~K}_{i}, \mathrm{y}_{A_{i}}\right)} \mathrm{K}_{i}{ }^{K_{i}}(\bmod p)$ is established.

2) signature generation process

Each agent signer $B_{i}$ of a random number $r \in Z_{q}^{*}$, calculated:

$$
\begin{aligned}
& R_{i}=g^{r_{i}}(\bmod p), R=\prod_{i=1}^{L} R_{i}(\bmod p), \\
& S_{i}=e_{i} h(m)-x_{B_{i}} y_{B_{i}} h(m, R)-r_{i} R(\bmod q) ;
\end{aligned}
$$

calculated: $R=\prod_{i=1}^{L} R_{i}(\bmod p)$;

Inspection equation:

$$
\left(y_{A_{i}}^{h\left(w_{i}, K_{i}, y_{A_{i}}\right)} K_{i}^{K_{i}}\right)^{h(m)}=g^{s_{i}} R_{i}^{R} y_{B_{i}}^{{ }_{B_{i}} h(m, R)}(\bmod p),
$$

is established, calculate: $s=\sum_{i=1}^{L} s_{i}(\bmod q)$,

The message of the proxy multi-signature is:

$$
\begin{aligned}
& \left(R ; K_{1}, K_{2}, \ldots, K_{L} ; w_{1}, w_{2}, \ldots, w_{L} ;\right. \\
& \left.s ; y_{A}, y_{A_{2}}, \ldots, y_{A_{L}} ; y_{B_{1}}, y_{B_{2}}, \ldots, y_{B_{L}}\right) .
\end{aligned}
$$

\section{3) Signature Verification Process}

Certifier $V$ After receiving the message $m$ and proxy multisignature, verify the identity of $m$ and all the proxy signer is within the set of all commission license signature $\left(w_{1}, w_{2}, \ldots, w_{L}\right)$

range, verify:

$$
\left[\prod_{i=1}^{L}\left(y_{A i}{ }^{\mathrm{h}\left(\mathrm{w}_{i}, \mathrm{~K}_{i}, \mathrm{y}_{A_{i}}\right)} \mathrm{K}_{i}{ }^{\mathrm{K}_{i}}\right]^{h(m)}=\mathrm{g}^{\mathrm{S}} \mathrm{R}^{R}\left(\prod_{i=1}^{L} y_{B_{i}}^{y_{B_{i}}}\right)^{h(m, R)}(\bmod \mathrm{p})\right.
$$

is satisfied.

\section{4) To resist the attack process}

We have 3.1 people for any signature forgery attack, for example, to attack the improved signature scheme, similar to the previous attacks, can be drawn:

$$
\begin{aligned}
& r_{1}=h(\mathrm{~m}, \mathrm{R}) \cdot\left(\mathrm{h}^{\prime}\left(\mathrm{m}^{\prime}, \mathrm{R}\right)\right)^{-1}(\bmod q), \\
& r_{2}=\left(1-r_{1}\right) h\left(\mathrm{~m}^{\prime}, \mathrm{R}\right) \cdot\left(\mathrm{h}\left(\mathrm{m}^{\prime}\right)\right)^{-1}(\bmod q), \\
& r_{3}=h(\mathrm{~m}) \cdot\left(\mathrm{h}^{\prime}\left(\mathrm{m}^{\prime}\right)\right)^{-1}(\bmod q) .
\end{aligned}
$$

So you want a successful attack, the need to find such a $\left(\mathrm{y}_{A_{1}}, K_{1}{ }^{\prime}\right)$, satisfies:

$$
\begin{aligned}
& \prod_{i=1}^{L}\left(y_{A_{0}}{ }^{h\left(w_{i}, k_{i}, y_{A_{i}}\right)} \cdot K_{i}^{K_{i}}\right)^{r_{3}}\left(\prod_{i=1}^{L} y_{B_{i}}{ }^{{ }_{B_{i}}}\right)^{r_{2}}= \\
& \left(y_{A_{o}}{ }^{\prime}\right)^{h\left(w_{1}, K_{1}, y_{A_{1}}\right)}\left(K_{1}\right)^{K^{\prime}} \cdot \prod_{i=2}^{L}\left(y_{A_{i}}{ }^{h\left(w_{i}, K_{i}, y_{A_{i}}\right)} K_{i}^{K_{i}}\right) \bmod p .
\end{aligned}
$$

But it is theoretically impossible, because you want from the following equation where:

$$
\begin{aligned}
& \left(K_{1}{ }^{\prime}\right)^{K_{1}{ }^{\prime}}=y_{A_{0}}{ }^{h\left(w_{1}, K_{1}, y_{A_{0}}\right)}\left(K_{1}\right)^{K_{1}} \bullet \\
& \prod_{i=1}^{L}\left(y_{A_{0}}{ }^{h\left(w_{i}, k_{i}, y_{A_{i}}\right)} \cdot K_{i}{ }^{K_{i}}\right)^{r_{3}-1} \cdot\left(\prod_{i=1}^{L} y_{B_{i}}{ }^{y_{B_{i}}}\right)^{r_{2}} \bmod p .
\end{aligned}
$$

Solving the $K_{1}^{\prime}$ process than the theoretically calculated discrete logarithm is even more difficult (because $K_{1}^{\prime}$ in the equation itself acts as this element, its computational complexity is greater than $O\left(\log (n)^{2}\right)$, and public key $y_{A_{o}}$ becomes $y_{A_{o}}$ 'is the formula established in the calculation of hash function (because $h(\bullet)$ is a one-way hash function collisions), so in the theory, the attack is failure.

In 3.2, we want to attack to be successful, you need to find such a $\left(\mathrm{y}_{B_{1}}{ }^{\prime}, K_{1}{ }^{\prime}\right)$, satisfies: 


$$
\begin{aligned}
& \prod_{i=1}^{L}\left(y_{B_{1}}{ }^{h\left(w_{i}, k_{i}, y_{B_{i}}\right)} \cdot K_{i}^{K_{i}}\right)^{\gamma_{3}}\left(\prod_{i=1}^{L} y_{B_{i}}{ }^{y_{B_{i}}}\right)^{2}= \\
& \left(y_{B_{1}}{ }^{\prime 2}\right)^{h\left(\mathrm{w}, K_{1}{ }^{\prime}, y_{B_{1}}{ }^{\prime}\right)}\left(K_{1}\right)^{K_{1}} \cdot \prod_{i=2}^{L}\left(y_{A_{i}}{ }^{h\left(w_{i}, K_{i}, y_{B_{i}}\right)} K_{i}^{K_{i}}\right) \bmod p .
\end{aligned}
$$

In 3.3, we want to attack to be successful, you need to find such a $\left(\mathrm{y}_{C_{0}}{ }^{\prime}, K_{1}{ }^{\prime}\right)$, satisfies:

$$
\begin{aligned}
& \prod_{i=1}^{L}\left(y_{C_{0}}{ }^{h\left(w_{i}, k_{i}, y_{C_{i}}\right)} \cdot K_{i}^{K_{i}}\right)^{r_{3}}\left(\prod_{i=1}^{L} y_{C_{i}}{ }^{{ }_{C_{i}}}\right)^{r_{2}}= \\
& \left(y_{C_{0}}{ }^{\prime}\right)^{h\left(w, K_{1}, y_{C 0}{ }^{\prime}\right)}\left(K_{1}{ }^{\prime}\right)^{K_{1}{ }^{\prime}} \cdot \prod_{i=2}^{L}\left(y_{A_{i}}{ }^{h\left(w_{i}, K_{i}, y_{C_{i}}\right)} K_{i}^{K_{i}}\right) \bmod p .
\end{aligned}
$$

Therefore, similarly to $3.1,3.2$ and 3.3 for the attacks in the calculation of the time and complexity of computing discrete logarithms is over, so the model can be modified to resist a general attack.

\section{5) Security Analysis and Discussion}

Since the introduction of the new program as well as increased commission parameter, its security has been enhanced, specifically as follows:

(1) new programs, including a proxy signature proxy signer's information, as well as others such as authorization and so can not pretend to participate in proxy multi-signature process, nor can the legitimate proxy multi signature repudiation.

(2) The new program introduced a letter of appointment, and for each, can be limited by the ability for the signature power of attorney can be used are constrained proxy signature and a certain period of the valid range, it can not be beyond the competence of signature, to avoid the abuse of the rights of proxy signature.

(3) can be set through the development of a legal proxy predecessors, beyond the signature others designated muster areas such participation may reject to accept, therefore, even if sent to other people, if not within the scope of the previous formulation of the agent then, is not the representative to carry out multi-signature process.

Through the above analysis we can see, the new proxy multi-signature scheme can satisfy the following properties:

(1)Verifiability: any certifier can verify this since the proxy multi-signature is valid and can be confirmed whether or not all of the original signers recognize these documents have been signed in accordance with a valid signature.

(2)Can not be forged strong line: Because proxy multisignature includes proxy signer's information, so in addition to anyone who thought the signature can not be substituted for forged signature, signature overcome denial.

(3)Proof of identity of the strong: because the proxy multisignature includes a proxy signer's public key, so anyone can participate in the proxy signature confirmation process signer from the proxy signature inside.

(4)A strong non-repudiation: Once a proxy signer to participate and generate a valid proxy signature multi-after, we can not deny.

(5)To prevent the abuse Signature: Signature proxy multisignature process ensures that if strictly followed all of the original signers of the commission information generation.

(6)Efficiency of the new signature program: the new program compared with the original program adds some parameters, but in contrast to the original scheme operation only increased the number of four operations, a new program on the operation efficiency and space utilization with the original plan remained unchanged.

\section{CONCLUSION}

Based on the basis of research and analysis of Zhou Hongsheng[7]et al paper, combining the characteristics of proxy multi-signature, the second proxy multi-signature scheme given in the original text is analyzed and attack, discovered its existence in security deficiencies. After the process of signature of the principal,the signature generation process and signature verification process of revision, the parameter is added on the basis of the original,and it is proved that the modified model in resisting attacks can be more complicated than computing discrete logarithm,so it can ensure the security of the signature.Proxy multi-signature and application affect people's production and life in the relevant fields have a positive role in promoting the same time, issues related to the efficiency of its processes and its signature and other security is the need for more in-depth study and discussion.

\section{REFERENCES}

[1] Mambo M, Usuda K, Okamoto E. Proxy signatures Delegation of the power to sign messages[J]. IEICE Trans Fundamentals, 1996.E79A(9):1338-1353.

[2] Boyd C.Multi-Signatures Revisited[A]. Cryptography and Coding III[C] Oxford:Clarendon Press 1993:21-30.

[3] Ham I.Digital Multi-Signature with Distinguished Signing Authorities[J] Electronics Letters, 1999,35(4):294-295.

[4] Yi L,Bai G,Xiao G. Proxy multi-signature scheme A new type of proxy signature scheme[J]. Electronics Letters, 2000:527-528.

[5] 傅晓娅,伊丽江,肖国镇. 一类行的代理多重签名体制 [J].西安电子科技 大学学报,2001,28(6): 729-731.

[6] 程元元,毛志芹. 基于代理多重签名的电子公文交换系统研究 [J]. . 中 州大学学报, 2014,31(5).116-120.

[7] 周红生,铁玲, 李建华, 倪佑生. 一类霄强的代理多重签名体制 [J]. . 上海交通大学学报, 2004,38(1):83-86. 